\title{
GSM Based Security Lock Using Linear Actuator
}

\author{
Y. N. Ravindra, R. B. Konda
}

Department of Electronics, Smt. V.G. College for Women, Kalaburagi, Karnataka, India

\begin{abstract}
Security describes the protection of life and property. There are doors to keep people out, key locks and chain reinforce the mode of security. Doors are being made of metals not wood anymore. Influential persons in our society have bullet proof doors to ensure a good measure of security of self and family. This has brought about the need to review the reliability of already existing systems and look into the possibility of creating better system that are smarter and more secure. The existing system largely consists of physical lock and keys. The problem with existing system is that it can cause security issues with the case of burglaries. The burglar can grapple with vault key and study it which can cause considerable damage to the property and valuable materials in the safe. Also, physically locks can be opened by lock picks. The main idea of designing the GSM based security locking system is to provide many modern security features than manual operated mechanical lock. The proposed locking system uses Microcontroller, GSM modem, switching circuit, relay and Linear Actuator.
\end{abstract}

Keywords: Microcontroller AT mega 16, Linear Actuator, GSM mode, Relays

\section{INTRODUCTION}

Today it is essential to provide the security system employing various sensors and alarm system in residential communities. A secure wireless alarm system for residential and other applications had been developed which incorporates central monitoring system for continuous monitoring the status of various sensors which are placed at the target place. In this proposed work idea of designing the GSM based security locking system is to provide many modern security features. When authorized user whose mobile number has been stored in the microcontroller dial the mobile number of the SIM card which is inserted in the SIM try of the GSM modem, after couple of rings the call has been disconnected by the microcontroller, once it matched with the stored number in the microcontroller. Then microcontroller output pin of the port D provides the high output, which in turn drive the relay, the relay switches the linear actuator so door is made to lock. Once the door locked, then return call back from GSM modem to user mobile number acknowledge the door lock. If user wants to unlock the door, once again user has to dial the mobile number of the SIM card, microcontroller receives the call, compare with the stored number and switches the actuator which in turn unlock the door. If any person whose mobile number has not been stored in the microcontroller fails to activates the lock. User only get return call while locking the door and not during unlocking the door.

\section{BLOCK DIAGRAM AND DISCRIPTION}

As shown in the Fig. 1. Microcontroller block uses AVR microcontroller ATmega16. Which is high performance low power Atmel? AVR microcontroller 
and has advanced RISC Architecture, 16k bytes of insystem self-programmable flash program memory, This low power CMOS AVR core combines a rich instruction set with 32 general purpose working registers. All the 32 registers are directly connected to the Arithmetic logic unit (ALU), allowing two independent registers to be accessed in one signal instruction executed in one clock cycle. The resulting

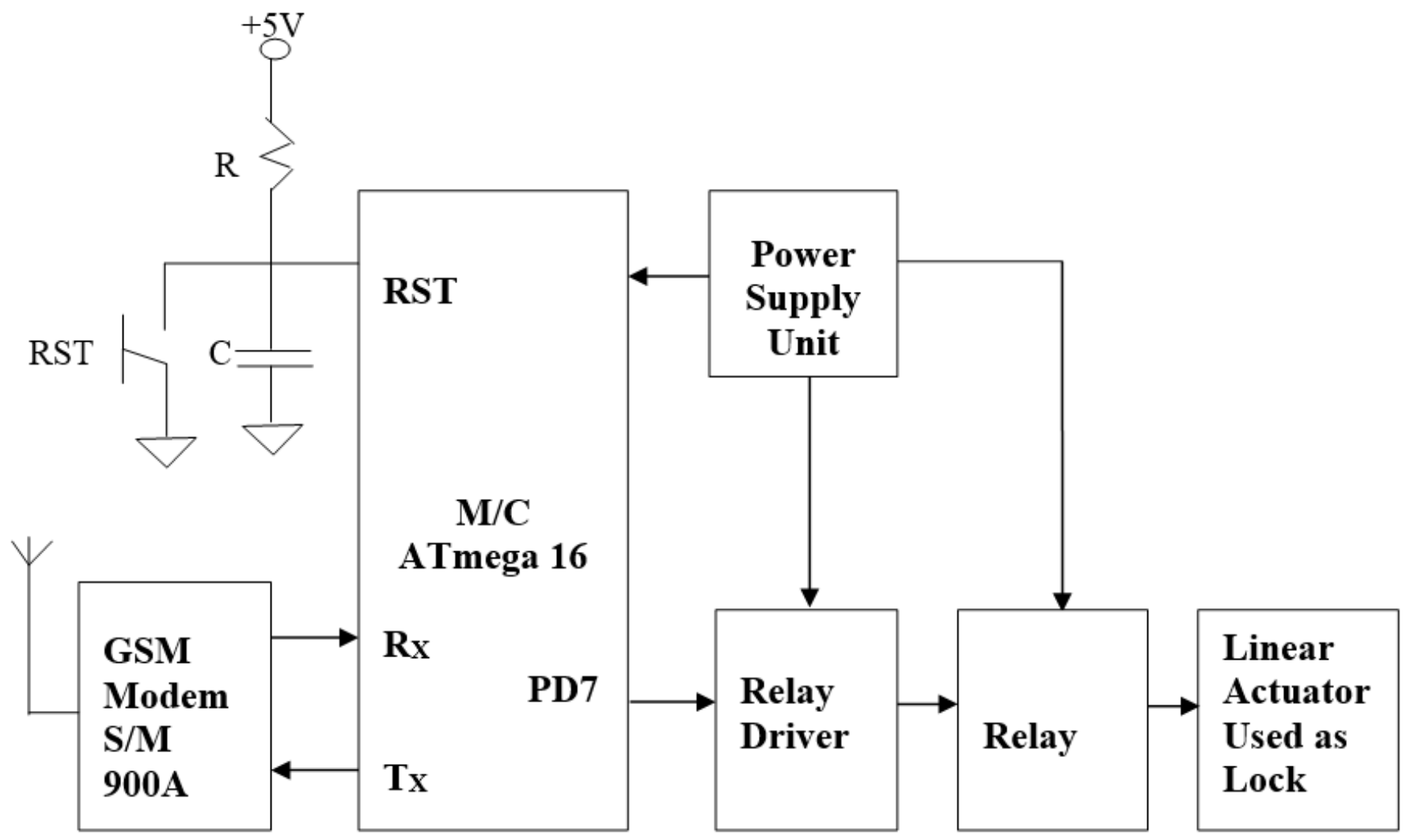

Figure 1: Block Diagram of GSM based Security Lock using Linear Actuator

modem one can make audio calls, SMS, attend the

The GSM modem block unit uses GSM modem SIM900A. GSM/GPRS modem RS232 is built with dual band GSM/GPRS engine. SIM 900A, works in frequencies $900 / 1800 \mathrm{MHz}$. the modem is coming with RS232 interface, which allows to interface with PC or microcontroller. The band rate is configurable from 9600-115200 through AT command. The GSM/GPRS mode is having internal TCO/IP stack to enable to connect with internet is a GPRS. It is suitable for SMS, voice as well as DATA transfer application. The onboard regulated power supply allows to connect wide range of unregulated power supply. Using the architecture is more code efficient while achieving through puts up to ten times faster than conventional CISC microcontroller. These AVR microcontrollers are better on noise immunity and work good in industry conditions such as noise, humidity, temperature and vibration. incoming calls and internet at through simple AT commands.

\section{A. Features}

- Dual band GSM/GPRS 900/1800 MHz

- Configurable band rate

- Built in network status LED

- Inbuilt powerful TCP/IP protocol stack for internet data transfer over GPRS

- SIM card holder

- Low power consumption $1.5 \mathrm{~mA}$ (sleep mode) 
The relay driver block consists of transistor amplifier to boost the current level of the output current provides by the microcontroller and provide sufficient current to drive the relay. The relay block uses electromagnetic relay which is used to switch the Linear Actuator ON/OFF with the help of relay driving transistors. It also provides voltage isolation between low control circuit and high Linear Actuator operating voltage and avoids electric shock. The proposed system uses Linear Actuator as safety Lock, which is linked to the system to be secured. This Linear Actuator is controlled by the relay.

\section{B. Working}

The Microcontroller $(\mathrm{M} / \mathrm{C})$ is programmed in such a way that only authorized user can operate the system, which is done by storing user mobile number in microcontroller. So, when authorized user dial the mobile number of the SIM card of the GSM modem after two to three rings the call has been cut off, the $\mathrm{M} / \mathrm{C}$ received the number from GSM modem through proper AT command and compare with stored number if both matches, $\mathrm{M} / \mathrm{C}$ send high output (PD7) to the relay driver and relay which in turn activate the Linear Actuator and hence the security lock. After few second $\mathrm{M} / \mathrm{C}$ send the stored mobile number back to user through GSM modem acknowledging the activated lock. If user wants to unlock the system once again user has to call to the mobile number of the SIM card again after two to three rings the ring will be stopped, $\mathrm{M} / \mathrm{C}$ output deactivate the lock.

Unauthorized person whose mobile number has not been stored in the $\mathrm{M} / \mathrm{C}$ fail to activate the lock so, the system is made authorized to activate or deactivate the lock only that user whose mobile number has been stored in the $\mathrm{M} / \mathrm{C}$.

\section{Power Supply}

The unit needs $+5 \mathrm{~V}$ power supply for $\mathrm{M} / \mathrm{C}$ and associated circuits and $+12 \mathrm{~V},-12 \mathrm{~V}$ for Linear Actuator. The $+5 \mathrm{~V}$ power supply is constructed by using step down transformer 0-9V/0.5A, bridge rectifier formed by $1 \mathrm{~N} 4007$ diodes, filter capacitor $1000 \mathrm{mf} / 25 \mathrm{~V}$,voltage regulator IC 7805 and decoupling capacitor $100 \mathrm{mf} / 25 \mathrm{~V}$.

The bipolar power supply is constructed by using a step down transformer 12V-0-12V/1A, bridge rectifier using $1 \mathrm{~N} 5402$ diodes and filter capacitor $2200 \mathrm{mf} / 25 \mathrm{~V}$.This bipolar power supply is necessary to activate the Linear Actuator.

\section{Advantages}

1. Security lock can be operated from long distance

2. Economical design

3. Can be used by everyone

4. No need to have smart phone

5. No cost since it operates on missed call

6. The system assists handicapped/old aged persons

\section{E. Disadvantages}

The system is network dependent; hence network congestion can reduce the reliability of the system. It needs continuous power supply and costlier compare to mechanical lock.

\section{III.CONCLUSION}

The proposed work 'GSM Based Security Lock Using Linear Actuator' works satisfactorily. The unit is tested with valued SIM inserted in the SIM try of the GSM modem and operating range found to be very large. The present system uses missed call to switch the lock, so only one lock can be used means one cannot use more than one lock to switch door lock or door unlock. But by using SMS switching technique one can switch any number of lock, but switching lock by SMS may costs more so, in the present system the hardware has been implemented for only one lock. The proposed unit is the one of the example showing how one can use the Linear Actuator for locking 
application. This versatile system finds many applications in different sectors such as Schools, colleges, hotels, Banks, Industries, Scientific institutions etc.

\section{REFERENCES}

[1] Ushie James Ogri, Donatus Enang Bassey Okwong, Akaiso Etim, "Design and construction of Door Locking Security Sys- tem Using GSM", International Journal Of Engineering And Computer Science, Vol. 2 Issue 7, pp. 2235-2257, July 2013

[2] Yanbo Zhao, Zhaohui Ye, "A low cost GSM/GPRS based wireless home security system", Consumer Electronics, IEEE Transactions on Vol.54, Issue: 2, May 2008.

\section{Cite this article as :}

Y. N. Ravindra, R. B. Konda, "GSM Based Security Lock Using Linear Actuator", International Journal of Scientific Research in Science and Technology (IJSRST), Online ISSN : 2395-602X, Print ISSN : 23956011, Volume 7 Issue 2, pp. 356-359, March-April 2020. Available at doi : https://doi.org/10.32628/IJSRST207266 Journal URL : http://ijsrst.com/IJSRST207266 